environmental exposure to bisphenol A (BPA). This study aims to examine the association between nightshift work and prostate cancer risk among Hong Kong Chinese men after take into account more environmental exposures.

Methods We consecutively recruited 431 incident prostate cancer cases and age frequency matched 402 controls who had complete information on nightshift work. After receiving written consents, trained researchers interviewed participants using a standard questionnaire to obtain information on socio-demographics, smoking, dietary habits, habits of using plastic food containers, family cancer history, and occupational history and nightshift work. A newly developed novel cumulative BPA exposure index (CBPAI) was used to estimate chronic BPA exposure. Odds ratio and 95\% confidence interval (95\% CI) was performed using multiple logistic regression analysis.

Results The mean age of prostate cancer cases was comparable to the controls (69.4 vs 68.2 years). Compared with the controls (39.1\%), more cases were less educated with a higher proportion of 'primary school or below' (41.1\%). More cases than controls were the nightshift workers $(13.5 \%$ vs $7.5 \%)$. After adjustment of age and socioeconomic characteristics, the OR of nightshift work to prostate cancer was 1.87 (95\% CI: 1.16 to 3.01$)$, and the OR retained statistical significance $(\mathrm{OR}=1.76,95 \% \mathrm{CI}: 1.07$ to 2.89$)$ after environmental exposures mainly from dietary sources were further adjusted.

Conclusions Results from this study provided supportive evidence that there might be a link between nightshift work and prostate cancer. The main merit of this study is that more environmental risk factors were considered in quantifying the association.

Funding Health and Medical Research Fund (Ref. No. 11121091), Hong Kong Special Administrative Region, China.

\section{6d WHAT SHOULD WE TELL SHIFT WORKERS TO DO TO REDUCE THEIR CANCER RISK?}

Damien McElvenney. Institute of Occupational Medicine, Edinburgh, UK

\subsection{6/oemed-2018-ICOHabstracts. 1378}

Introduction In 2007, a working group of the International Agency for Research on Cancer classified shift work that involves circadian rhythm disruption as probably carcinogenic to humans. We carried out a review of the human epidemiological and mechanistic evidence, along with relevant health and safety policies and practice, to identify practical measures that should be undertaken to reduce the risk of cancer among shift workers.

Methods A literature search was carried out of the Proquest Dialogue databases, which includes MEDLINE, in order to identify the relevant scientific literature published from 2005-2015. In addition, we carried out an internet search for current health and safety policies and practices for managing shift workers.

Results There is evidence from the epidemiological studies that the increase in breast cancer risk amongst women who have worked night shifts is relatively modest and could be zero. Other risk factors for breast cancer associated with night work, such as poor diet, should be addressed by employers. Evidence for other cancers remains limited and inconsistent. If a mechanism exists, it is most likely to be night-time suppression of melatonin production. There has been limited evaluation of interventions to manage shift workers and research in this area is fragmented. Health and safety policies provide little on prevention.

Conclusion Health and safety practices for shift workers should address reduction of health risks by choosing shift rotations that minimise impact on circadian disruption, by employers facilitating female workers to attend breast cancer screening and by promoting and facilitating a healthy lifestyle.

\section{6e CURRENT PROGRESS AND STATE OF SCIENCE IN POPULATION STUDIES OF CIRCADIAN/SLEEP DISRUPTION ON CANCER RISK AND SURVIVAL, AND CIRCADIAN PHASE BIOMARKERS}

\begin{abstract}
1,2Schernhammer Eva. 'Brigham and Women's Hospital and Harvard Medical School, Harvard School of Public Health, Channing Division of Network Medicine, Boston, MA 02115, USA; ${ }^{2}$ Department of Epidemiology, Medical University of Vienna, Austria
\end{abstract}

\subsection{6/oemed-2018-ICOHabstracts. 1379}

Melatonin (5-methoxytryptamine) is an indoleamine produced primarily by the pineal gland, which is secreted exclusively during the dark phase of the light-dark cycle in humans. Several decades ago, reports indicated that melatonin possesses oncostatic properties, leading to novel hypotheses that diminished secretion of melatonin might promote the development of cancer. Growing evidence also demonstrates that visible light, including electric light, can acutely suppress melatonin production- a phenomenon often referred to as 'circadian disruption' particularly if it occurs at night, as commonly observed in shift workers.

In 2007, the International Agency for Research on Cancer classified shift work as a possible carcinogen, based on convincing experimental evidence and supportive, but still limited, epidemiologic data. Indeed, experimental data has consistently demonstrated that circadian disruption can promote carcinogenesis in animals; specifically, exposure to light at night and phase shifts in the light-dark cycle have accelerated tumour development in rodents. In humans, epidemiologic data continues to accumulate, with the majority of existing studies indicating that shift work is related to a modest increase in the risk of breast cancer. Initial studies have identified links between shift work and other cancers as well, although this evidence is very limited.

In this presentation, I will review epidemiologic studies of circadian disruption/sleep and cancer risk, including some preliminary studies on cancer survival, and with additional emphasis on circadian phase biomarkers (melatonin/cortisol).

\section{SHIFTWORK IN THE 21ST CENTURY: OLD AND NEW PROBLEMS AND APPROACHES}

${ }^{1}$ Frida Marina Fischer*, ${ }^{2}$ Stephen Popkin. 'Department of Environmental Health, School of Public Health, University of São Paulo. São Paulo, SP, Brazil; ${ }^{2}$ U.S. Department of Transportation, Volpe Centre, Cambridge, USA

10.1136/oemed-2018-ICOHabstracts. 1380

Aim of special session This session is expected to be interactive, providing the attendees with both an understanding of the social and physical harm than may come with working shifts, and an evaluation of different countermeasures developed to help ameliorate these problems. The question and answer portion is meant to be both 'ask the expert' for those 